\title{
Regioselectivity in the Addition of Vinylmagnesium Bromide to Heteroarylic Ketones: $\boldsymbol{C}$-versus $\boldsymbol{O}$-Alkylation
}

\author{
Carla Boga," Rayk Stengel, Rodolphe Abdayem, Erminia Del Vecchio, Luciano Forlani and \\ Paolo E. Todesco \\ Dipartimento di Chimica Organica “A. Mangini”, Viale Risorgimento, 4, 40136 - Bologna, Italy
}

\section{Supporting Information}

\section{Contents:}

General experimental details

S 1

${ }^{1} \mathrm{H}$ NMR spectrum of compound $\mathbf{2 5 a}$

S 2

${ }^{13} \mathrm{C}$ NMR spectrum of compound 25a

S 3

${ }^{1} \mathrm{H}$ NMR spectrum of compound 25d

S 4

${ }^{13} \mathrm{C}$ NMR spectrum of compound 25d

S 5

${ }^{1} \mathrm{H}$ NMR spectrum of compound 27a

${ }^{13} \mathrm{C}$ NMR spectrum of compound 27a

S 6

${ }^{1} \mathrm{H}$ NMR spectrum of compound $\mathbf{2 7} \mathbf{b}$

S 7

${ }^{13} \mathrm{C}$ NMR spectrum of compound $\mathbf{2 7 b}$

S 8

${ }^{1} \mathrm{H}$ NMR spectrum of compound $27 \mathrm{c}$

${ }^{13} \mathrm{C}$ NMR spectrum of compound 27c

S 9

S 10

${ }^{1} \mathrm{H}$ NMR spectrum of compound 27d

${ }^{13} \mathrm{C}$ NMR spectrum of compound $\mathbf{2 7 d}$

${ }^{1} \mathrm{H}$ NMR spectrum of compound 27e

$\mathrm{S} 13$

${ }^{13} \mathrm{C}$ NMR spectrum of compound $27 \mathrm{e}$

S 14

S 15

General: ${ }^{1} \mathrm{H}$ and ${ }^{13} \mathrm{C}$ NMR spectra were recorded at 300 or $400 \mathrm{MHz}$ and 75.46 or $100.56 \mathrm{MHz}$, respectively. Chemical shifts are referenced to internal standard TMS (for ${ }^{1} \mathrm{H}$ NMR in $\mathrm{CDCl}_{3}$ ) and to solvent (77.0 ppm for ${ }^{13} \mathrm{C} \mathrm{NMR} \mathrm{in} \mathrm{CDCl}_{3}$ ). $J$ values are given in $\mathrm{Hz}$. MS spectra were recorded at an ionisation voltage of $70 \mathrm{eV}$. Flash chromatography (FC) was performed on silica gel (0.040$0.063 \mathrm{~mm}$ ). Melting points are uncorrected. THF was distilled from sodium benzophenone ketyl. Air and moisture sensitive solutions and reagents were handled in a dried apparatus under an atmosphere of dry nitrogen. 


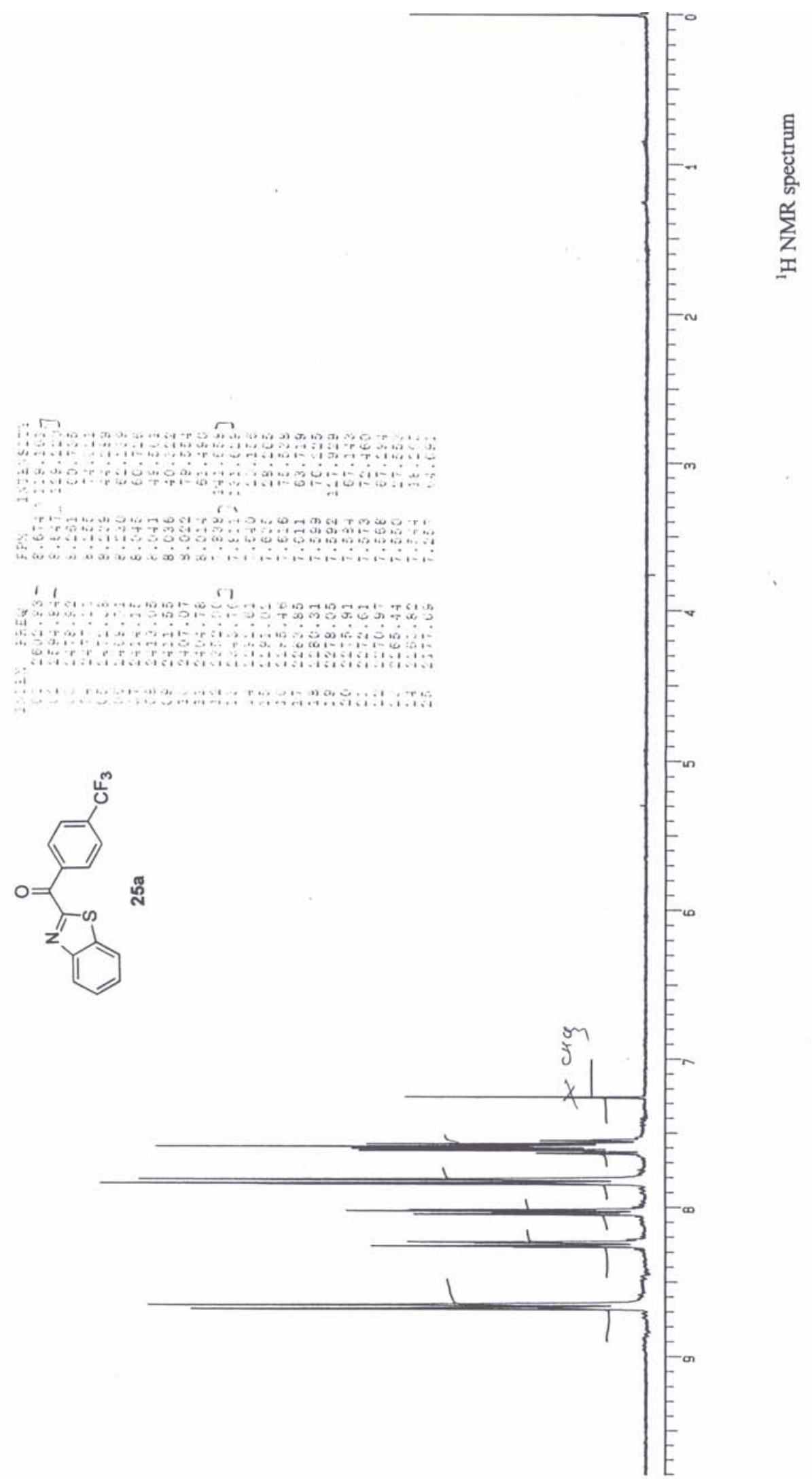



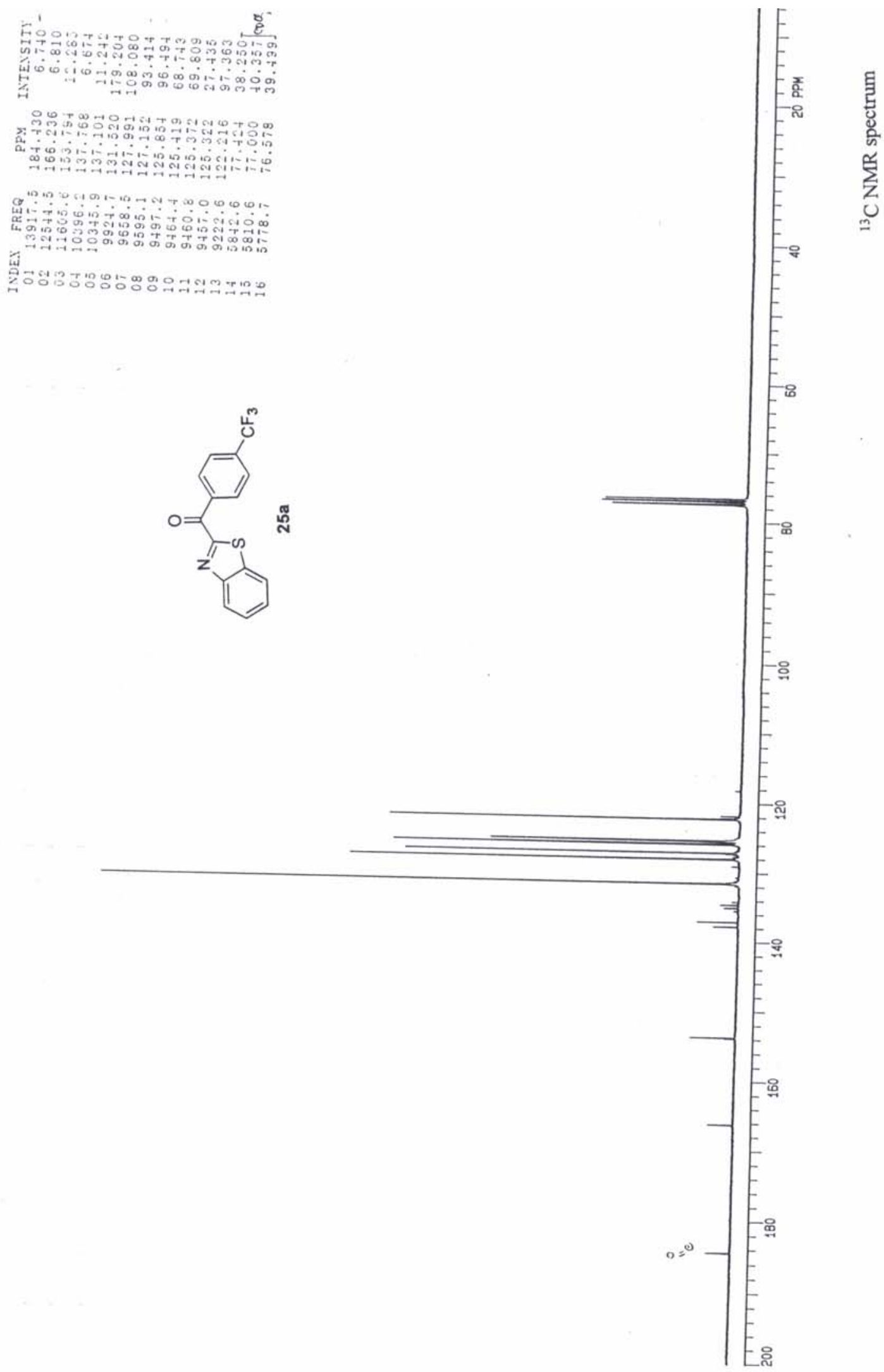


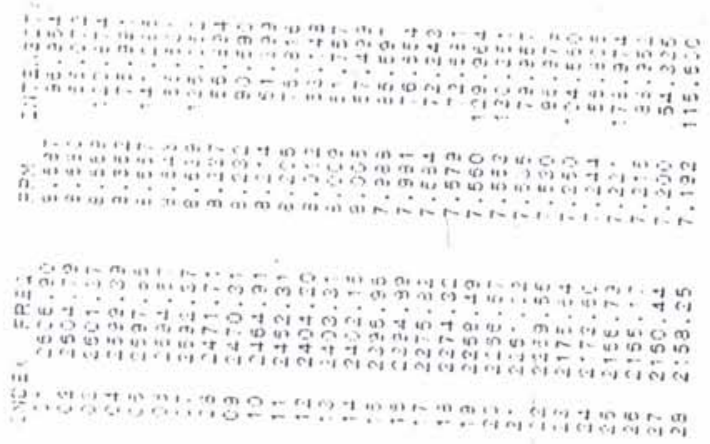

䍚<smiles></smiles>

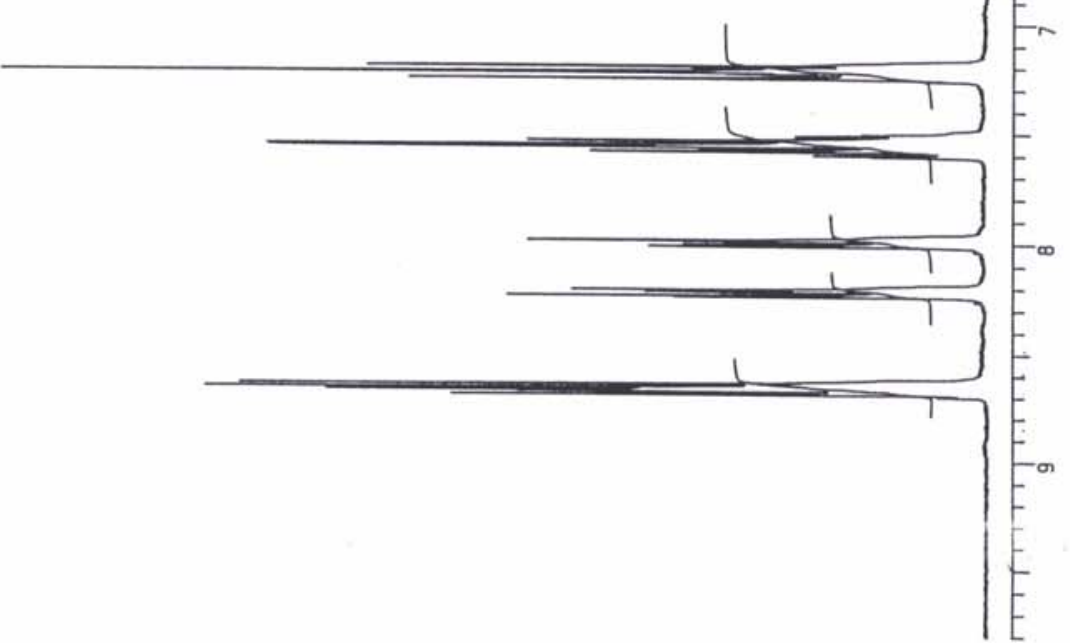



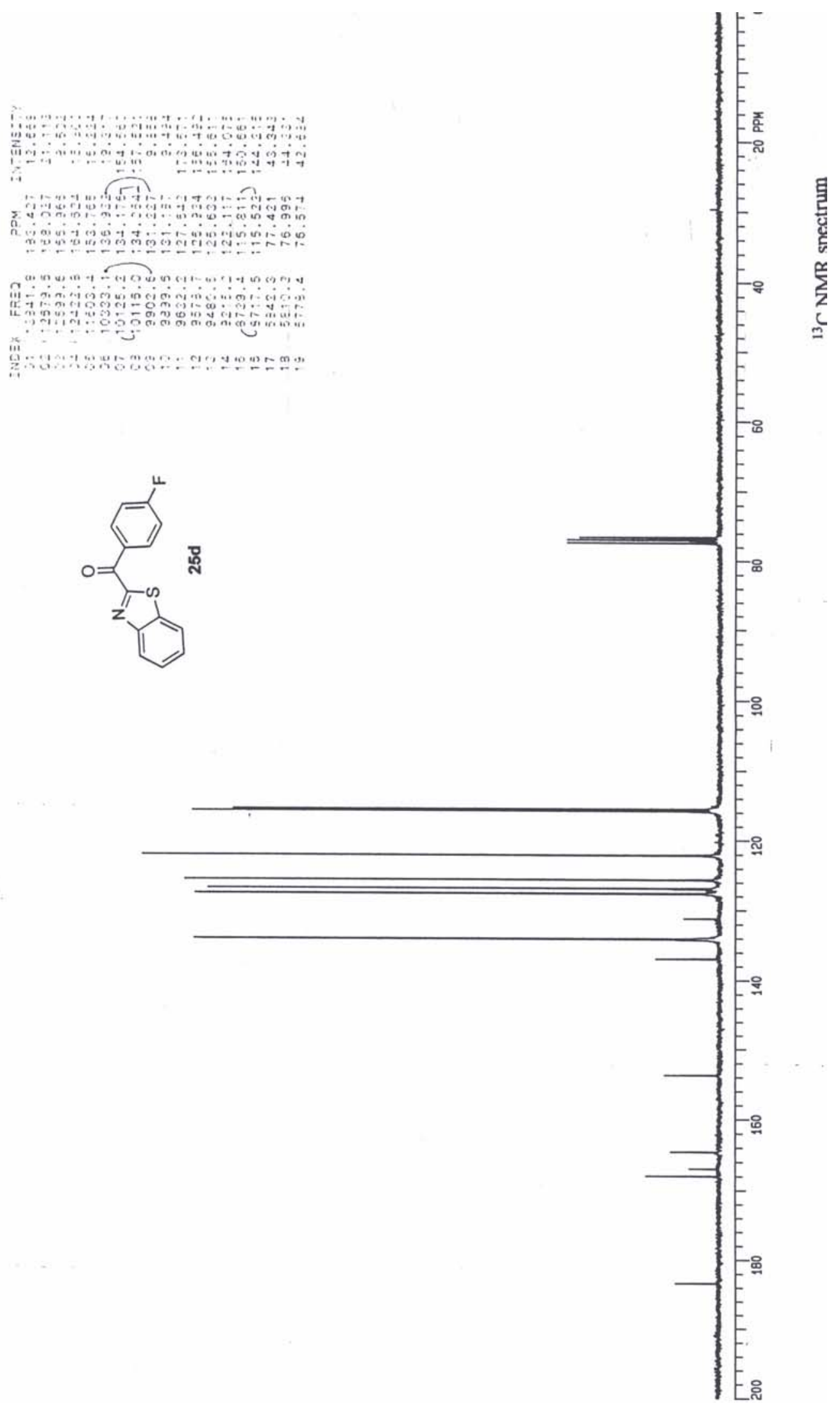

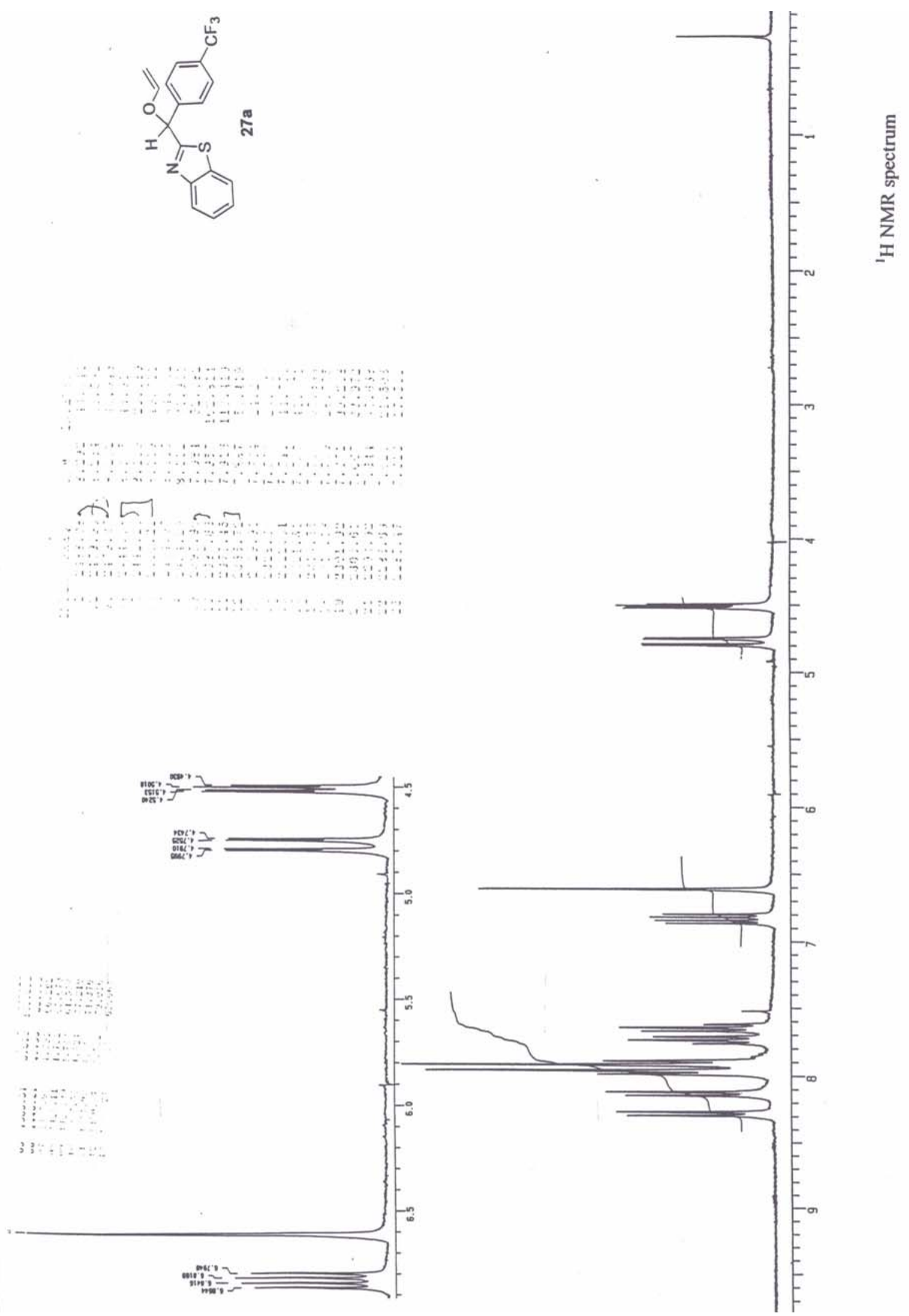

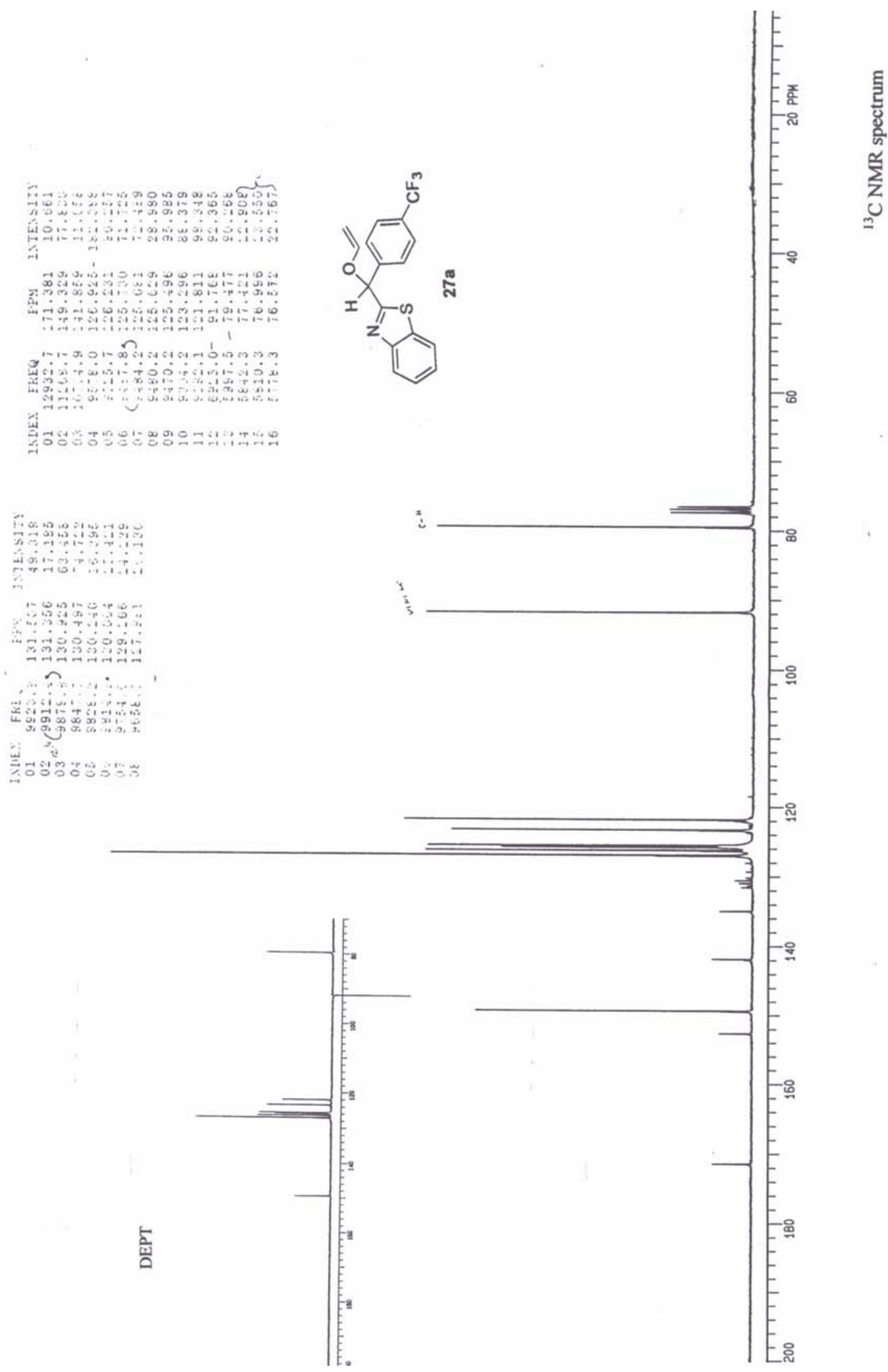


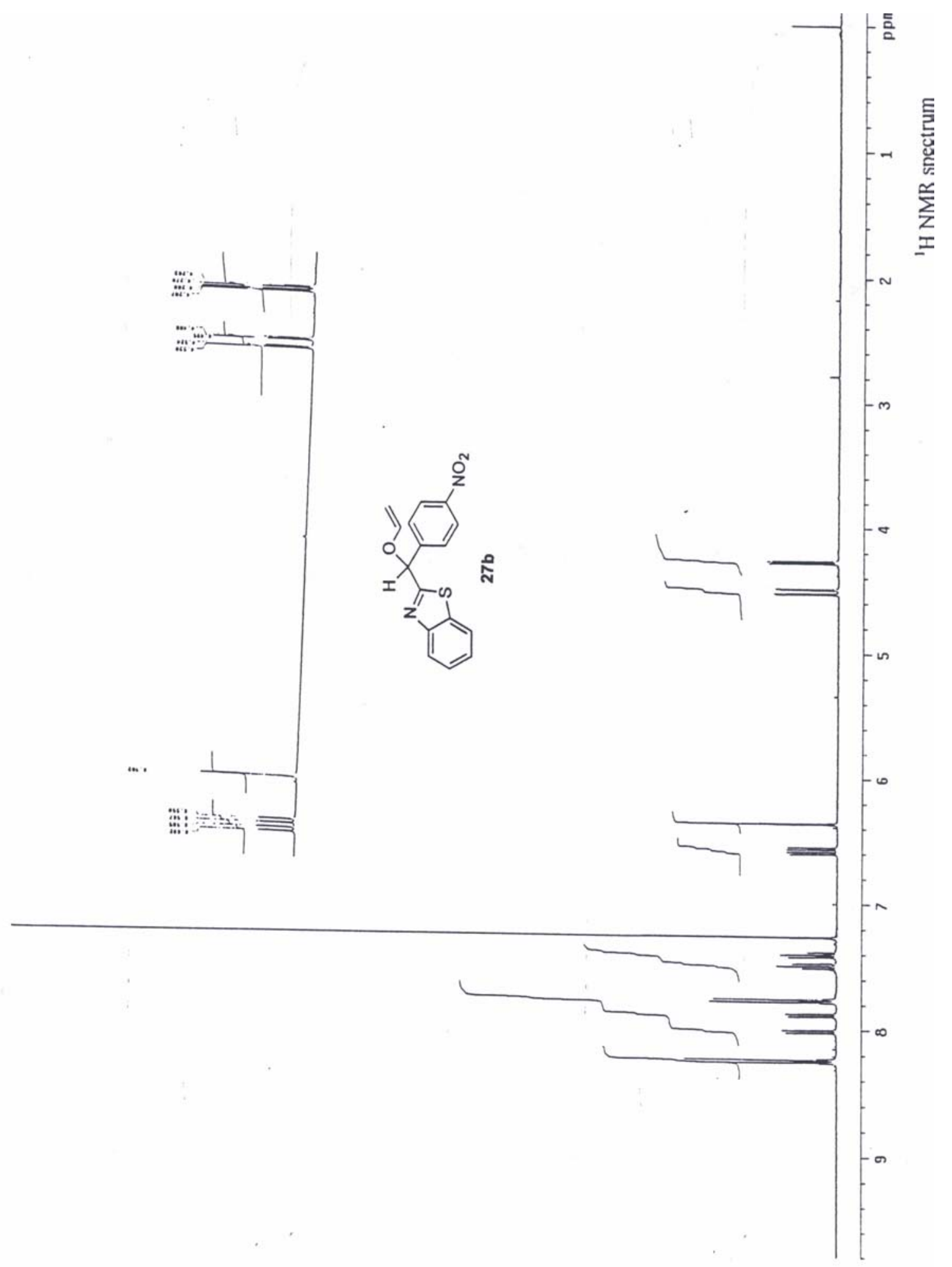




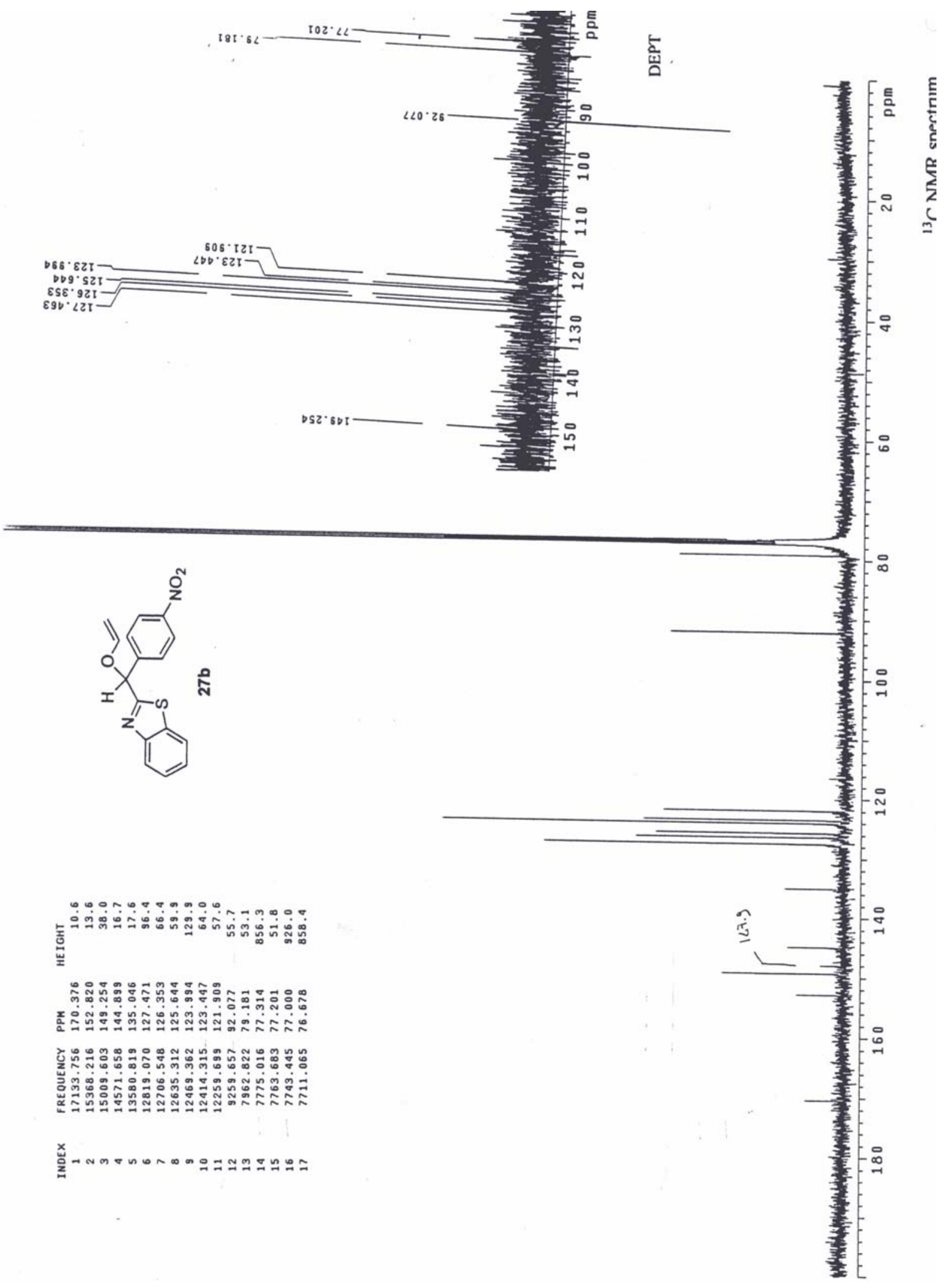



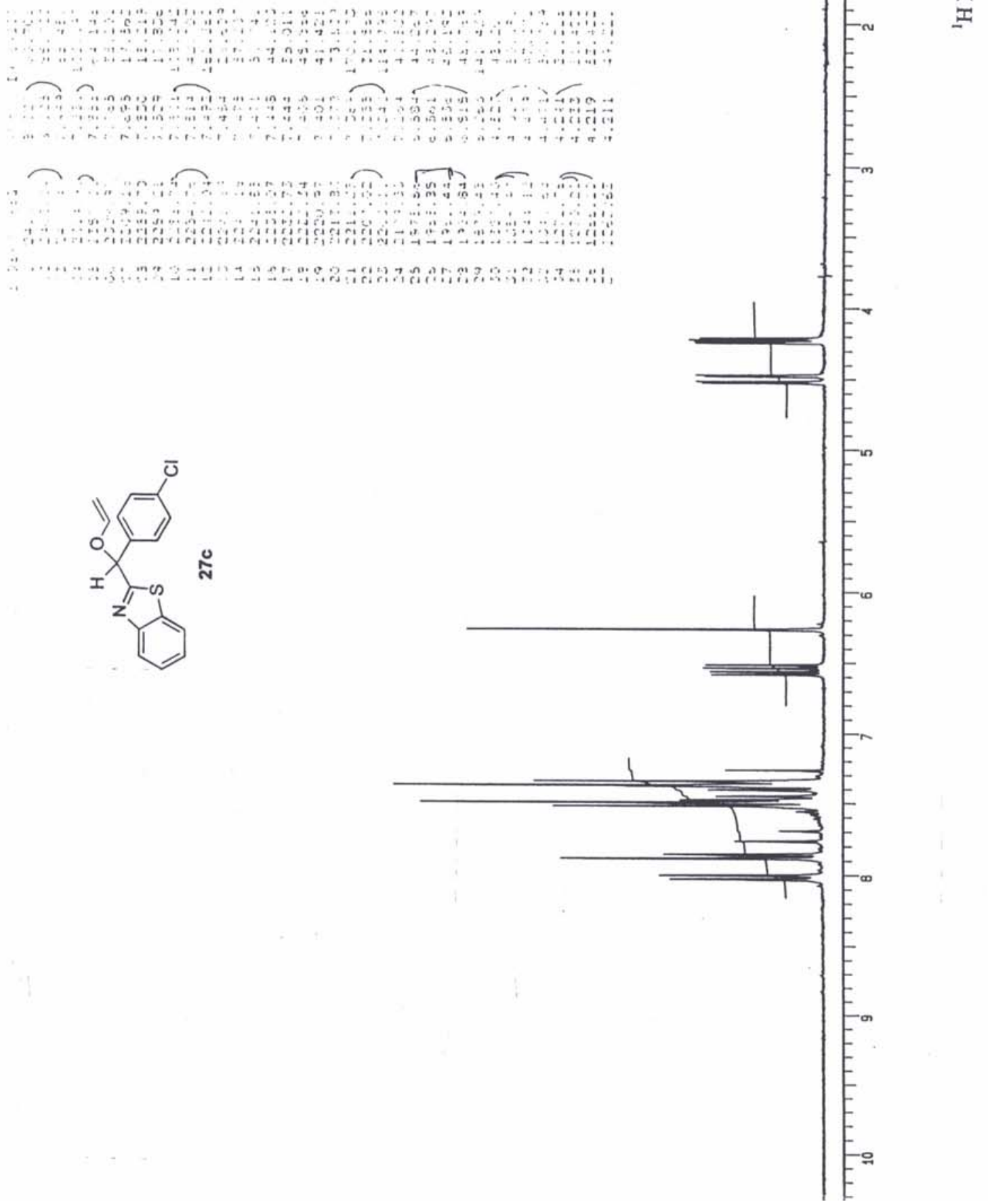


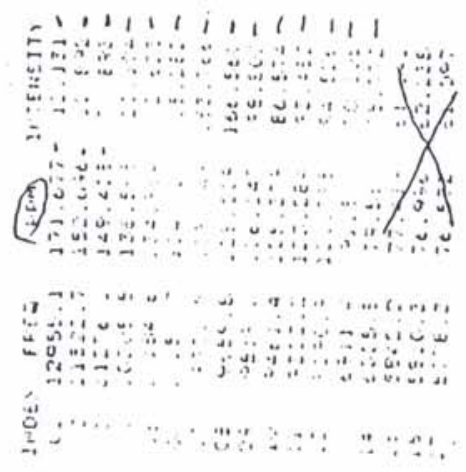

ह
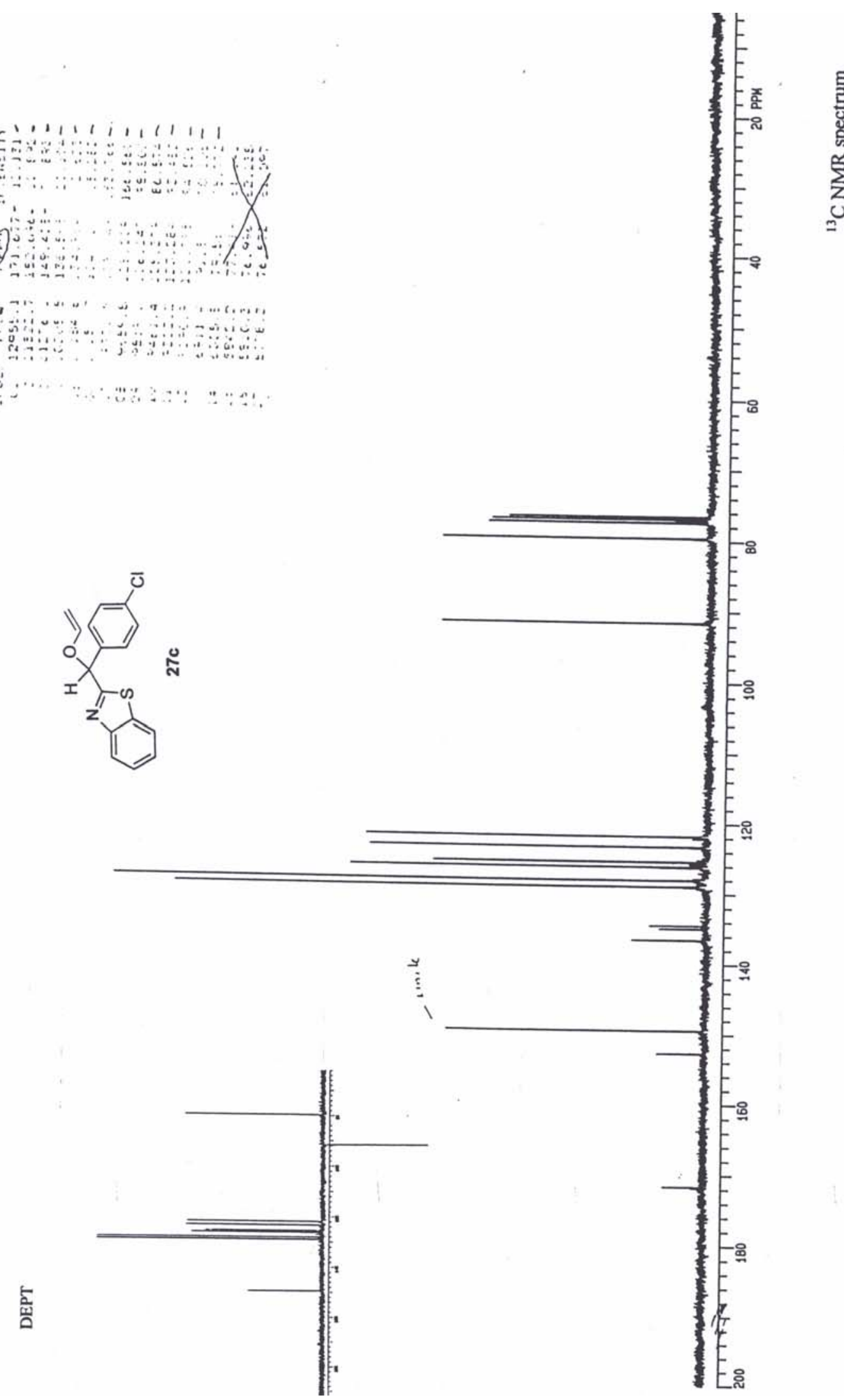


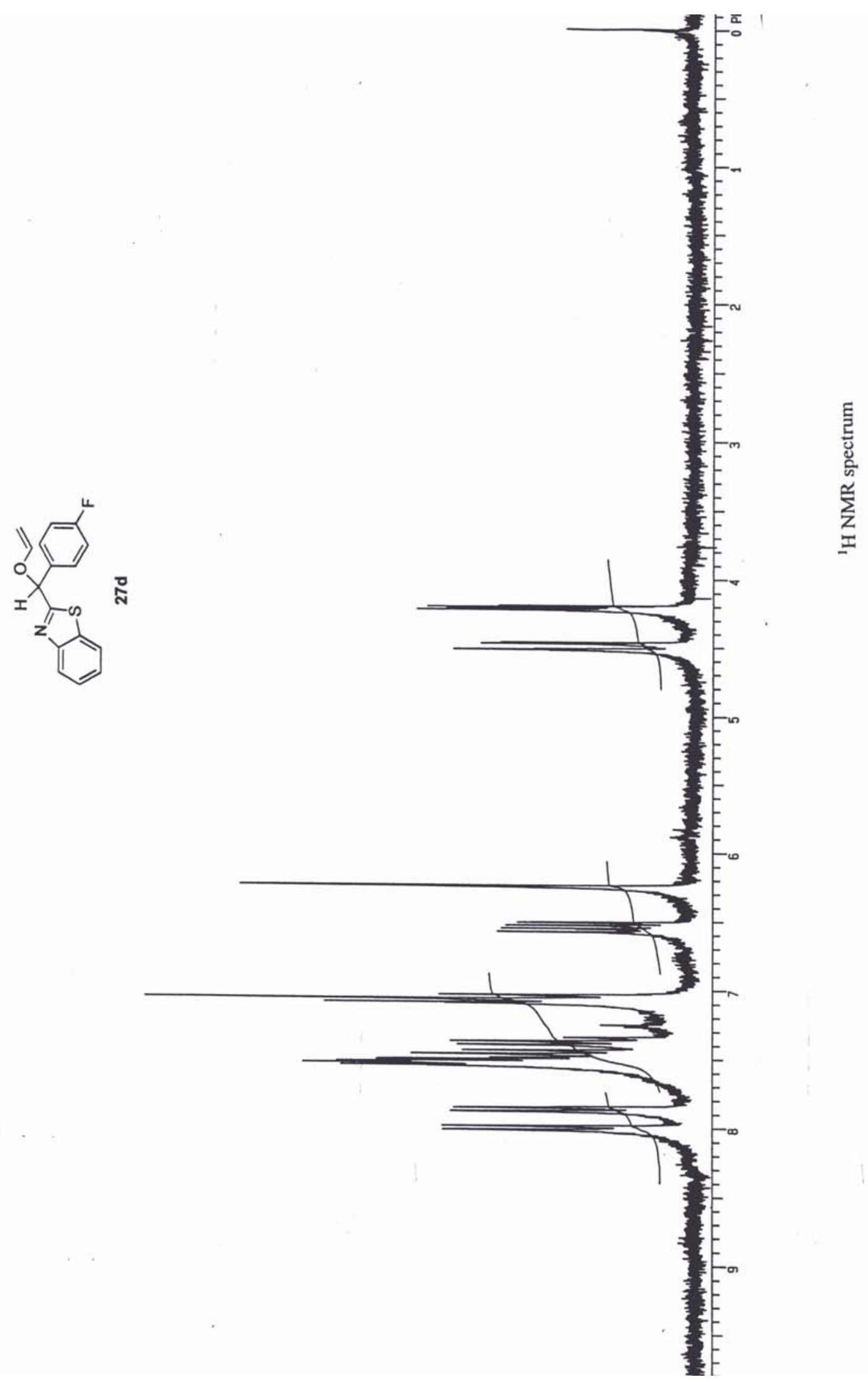




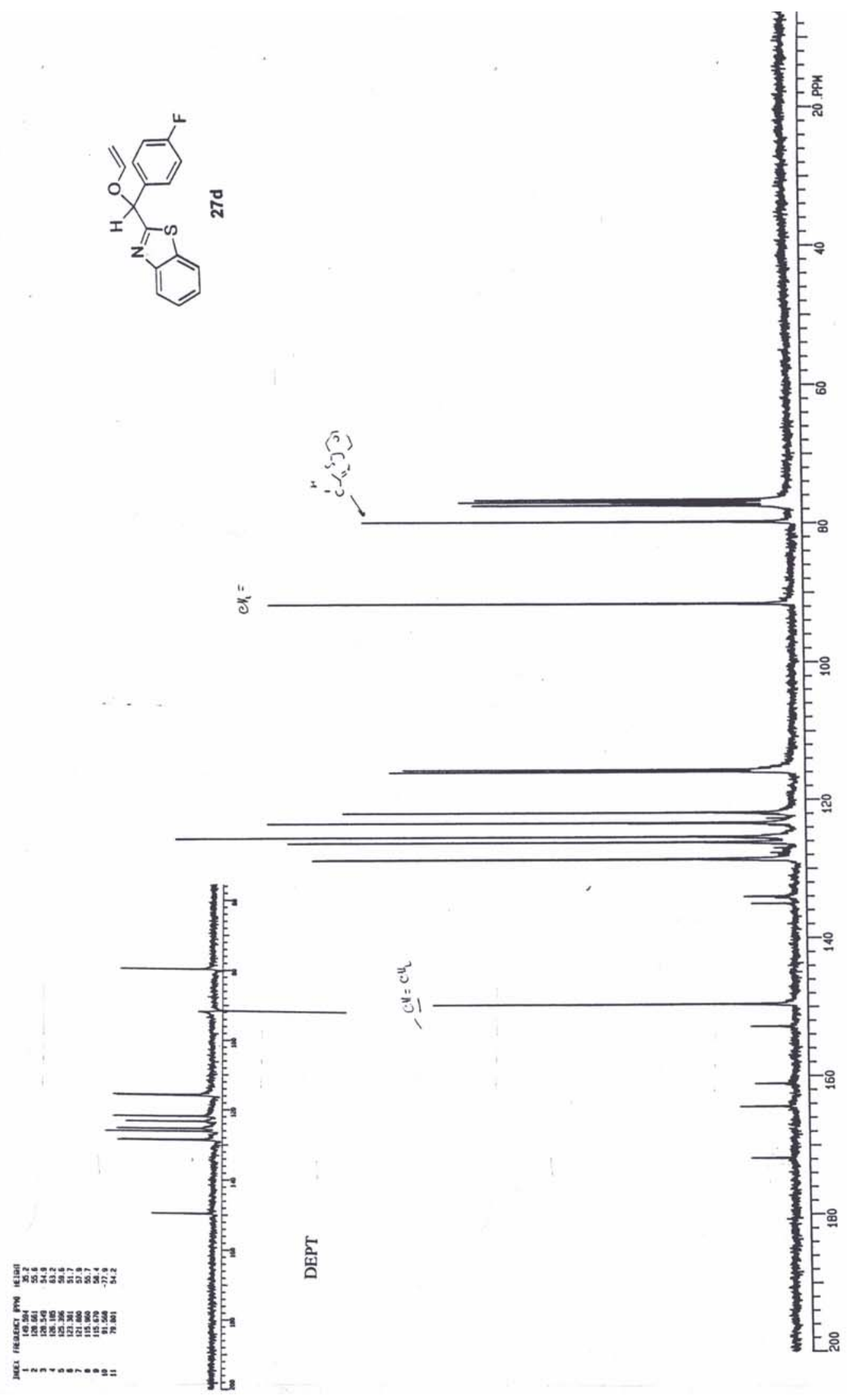




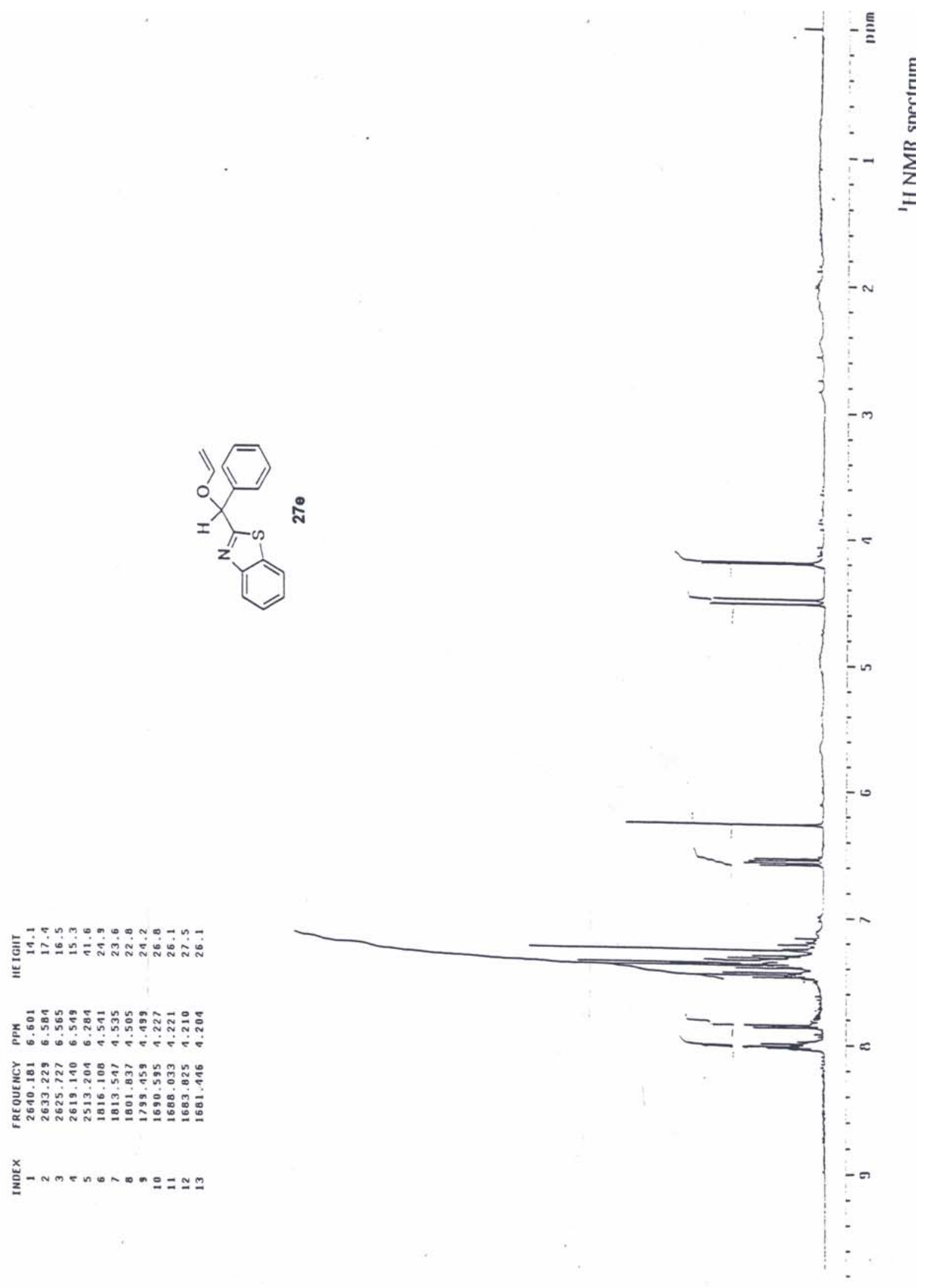




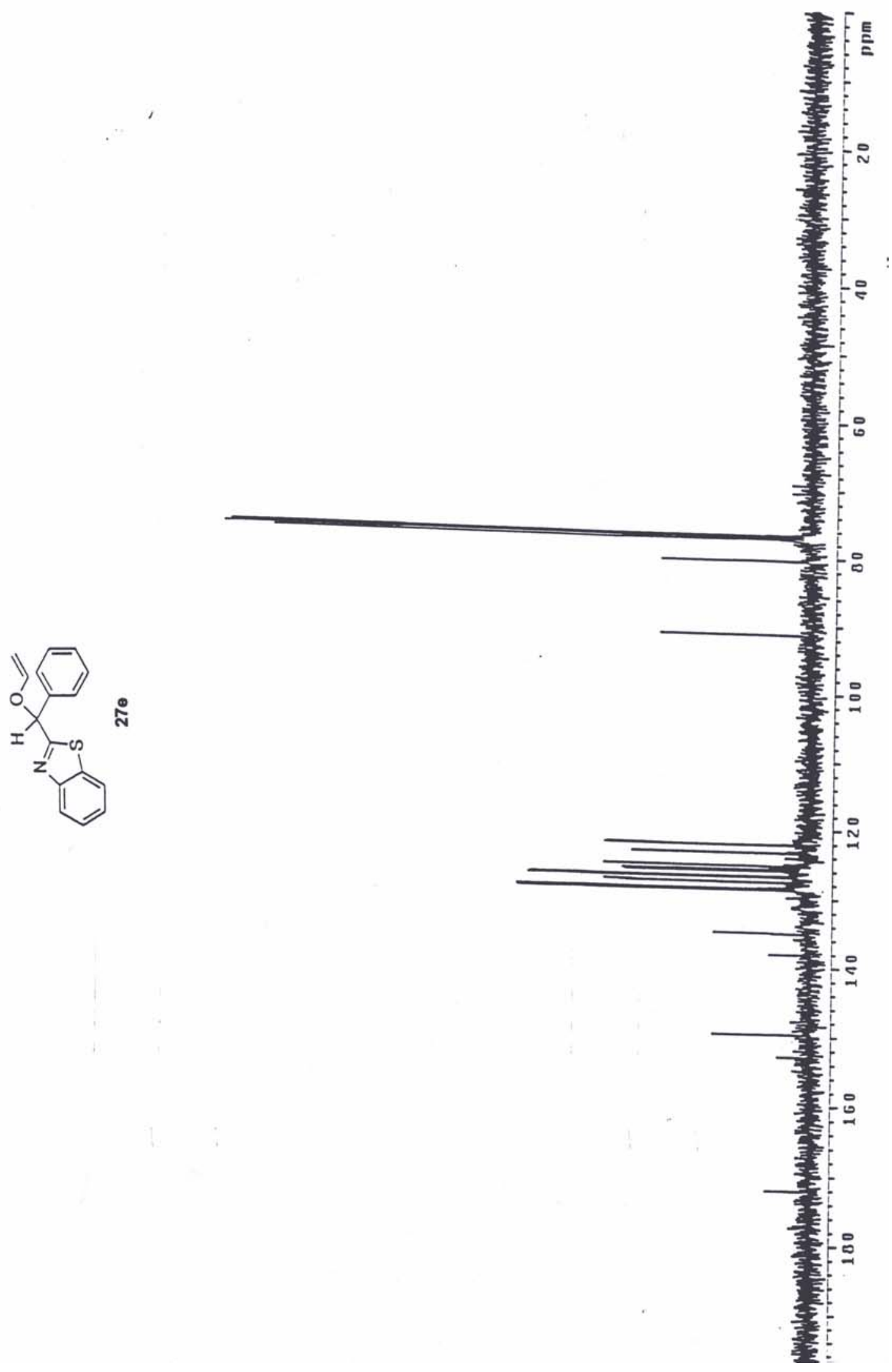

